\title{
LIFETRAC CODE FOR THE WEAK-STRONG SIMULATION OF THE BEAM-BEAM EFFECTS IN TEVATRON*
}

\author{
Dmitry Shatilov $^{\dagger}$, BINP SB RAS, Novosibirsk, 630090, Russia \\ Yuri Alexahin, Valeri Lebedev, Alexander Valishev, Fermilab, Batavia, IL 60510, USA
}

\begin{abstract}
A package of programs for weak-strong simulation of beam-beam effects in hadron colliders is described. Accelerator optics parameters relevant to the simulation are derived from beam measurements and calculations are made using OptiM optics code. The key part of the package is the upgraded version of the LIFETRAC code which now includes 2D coupled optics, chromatic modulation of betafunctions, non-Gaussian shape of the strong bunches and non-linear elements for beam-beam compensation. Parallel computations are used and in the case of the Tevatron ( 2 main IPs +70 parasitic IPs) the code has a productivity of $\sim 10^{10}$ particles $\times$ turns/day on a 32-node cluster of Pentium-IV $1.8 \mathrm{GHz}$ processors.
\end{abstract}

\section{INTRODUCTION}

Beam-beam effects are now one of the main limiting factors for the increase of the Tevatron luminosity [1]. Beambeam interaction causes particle losses and emittance blow up for both proton and antiproton beams, at injection and at the energy of experiment. Since the beams in Tevatron move in a common vacuum chamber, number of collision points is dramatically increased due to Parasitic Collisions (PC). With the present bunch pattern which has 3 trains of 12 bunches, besides the two main Interaction Points (IP) with head-on collision there are also 70 PCs. Parasitic Collisions act as nonlinear dipole correctors distorting the orbits of individual bunches and also shift their betatron tunes. An example of different bunch-to-bunch beam-beam behaviour is given in Fig.1. The so-called "scallops" (because of the distinctive shape of the plot) represent different blow up of the antiproton bunches in a train during first minutes after the beams are brought in collision.

All these facts suggest that for the detailed understanding of beam-beam effects in the Tevatron a complete computer model is required taking full account of the real (i.e. measured) optics features, including helical separated orbits, beta functions, coupling and collision order of bunches in the machine. To address this goal we created a set of computer codes capable of deriving machine parameters from the beam measurements, building a computer model of the machine, and simulating the beam-beam interaction. This report describes the technology of the computation. Two separate reports in these Proceedings present the detailed

\footnotetext{
* Work supported by the Universities Research Assoc., Inc., under contract DE-AC02-76CH03000 with the U.S. Dept. of Energy

$\dagger$ shatilov@inp.nsk.su
}

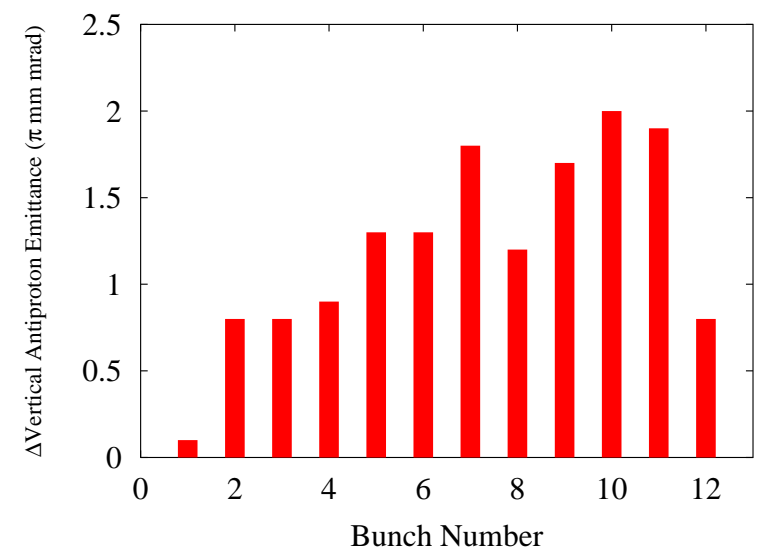

Figure 1: Bunch by bunch change of vertical antiproton emittance during first 15 minutes of the store \#3554.

description of the optics measurement at Tevatron [2], and discuss the results of simulations [3].

\section{BEAM-BEAM CODE}

Initially, the Beam-Beam code LIFETRAC was developed for simulation of the equilibrium distribution of the particles in circular electron-positron colliders [4]. In 1999 the new features have been implemented, which allowed simulating non-equilibrium distributions, for example proton beams. In this case the goal of simulations is not to obtain the equilibrium distribution but to observe how the initial distribution is changing with time. Number of simulated particles can vary in the range of $10^{3}$ to $10^{6}$, usually it is set to $(5 \div 10) \cdot 10^{3}$. The tracking time is divided into "steps", usually $10^{3} \div 10^{5}$ turns each. The statistics obtained during the tracking (1D histograms, 2D density in the space of normalized betatron amplitudes, luminosity, beam sizes and emittances) is averaged over all particles and all turns for each step. So, we get a sequence of frames representing evolution of the initial distribution.

The initial 6D distribution of macroparticles can be either Gaussian (by default), or read from a separate text file, prepared by another code (or manually). Besides, the macroparticles may have different "weights". This allows representing the beam tails more reliably with limited number of particles. Usually we simulate the Gaussian distribution with weights: particles initially located in the core region have larger weight while the "tail" particles with smaller weight are more numerous.

In the present bunch pattern ( 3 trains of 12 bunches) 
there are two main IPs and 70 PCs. When performing transformation through a main IP, the "strong" bunch is divided into slices longitudinally. The higher are the orders of significant betatron resonances which are supposed to make effect on the distribution, the greater must be the number of slices. In our simulations we used 12 slices in the main IPs where beta-functions are approximately equal to the bunch length and only one slice in PCs where beta-functions are much greater and we can neglect the betatron phase advance on the bunch length.

The transverse density distributions within "strong" slices are bi-Gaussian, allowing to apply the well-known formulae [5] for 6D symplectic beam-beam kick. However, a simple modification allowed simulating non-Gaussian strong bunches. Namely, the strong bunch is represented as a superposition of a few (up to three) Gaussian distributions with different betatron emittances. The kicks from all these "harmonics" are summarized additively. The calculation time is increased somehow (not very significantly) but the transformation remains $6 \mathrm{D}$ symplectic.

\section{TEVATRON OPTICS}

The Parasitic Collisions in Tevatron play a significant role in the beam dynamics. In order to account their contribution correctly we need to know the machine lattice of the whole ring, with all distortions, beta beatings, coupling, etc. First of all, we need to build a realistic model of the machine lattice, based on the real measurements. One of the most effective methods at the moment is based on measurement of the so-called differential orbits [2].

First, the closed orbit in Tevatron is excited using dipole correctors and corresponding orbit changes around the ring are recorded using the BPM system. The idea is to find gradient errors utilizing the fact that in the case of off-center orbit quadrupoles act as dipole correctors with the strength $\theta=K l \cdot x$, where $K l$ is the integrated quadrupole strength and $x$ is the orbit displacement.

If the initial closed orbit is excited using a single dipole corrector the displacement is described by a well known formula

$$
x(s)=\frac{\sqrt{\beta(x)}}{2 \sin (\pi \nu)} \theta \sqrt{\beta\left(s_{0}\right)} \cos \left(\left|\varphi(s)-\varphi\left(s_{0}\right)\right|-\pi \nu\right),
$$

where $\nu$ is the betatron tune, $\theta$ is the corrector kick, $\beta$ and $\varphi$ are the betatron function and phase, ans index 0 labels the corrector location.

Tevatron consists mainly of regular FODO structure. Since we are interested in the orbit displacements at quadrupoles where beta-functions are equal and phase advances are known $(\pi / 3$ per cell) the above formula can be rewritten as

$$
x_{m}=\frac{\beta_{f} \theta}{2 \sin (\pi \nu)} \cos \left(\frac{\pi}{3} m-\pi \nu\right) \approx \frac{\beta_{f} \theta}{2} \sin \left(\frac{\pi}{3} m\right) .
$$

Index $m$ numbers quadrupoles starting from 0 , where the dipole corrector is located $\left(\varphi\left(s_{0}\right)=0\right)$.
From this simplified analysis one can see that using a single dipole corrector it is possible to excite the orbit only in quadrupoles with indices $m \neq 3 k, k=0,1, \ldots$. Adding one corrector with $\varphi\left(s_{0}\right)=\pi / 3$ solves the problem. Hence, we come to a minimal set of four correctors, two in each betatron plane.

An additional orbit measurement involving change of the beam revolution frequency (or, equivalently, the beam energy) is intended to derive the dispersion function.

Then, we use the OptiM code [6] to build a model lattice corresponding to these measurements. Differential orbits computed by OptiM are fitted to the measured differential orbits by adjusting individual quadrupole and skewquadrupole strengths.

Both codes, OptiM and LIFETRAC, treat coupling using the same coupled beta-functions formalism [7]. The linear transport matrix between any two points can be derived from the coupled lattice functions and phase advances.

Actually, we built a "chain" of codes. Between OptiM and LIFETRAC there are two intermediate links. The first one is a perl script, which accepts the bunch number we are going to simulate, calculates the azimuths of PCs for that bunch, and extracts all the parameters (beta-functions, dispersions, phase advances, separations, crossing angles) for those azimuths from the very-long output file produced by OptiM. The second link reads these data, plus a separate "template" file with some specific tracking parameters, and produces an input file for LIFETRAC.

\section{Chromaticity}

Although we decided to use linear optics for the machine lattice model, there are two nonlinear lattice effects which we consider to be significant for beam-beam behaviour, and we included them into simulations. These are the chromaticities of beta-functions excited in the Main IPs and chromaticities of the betatron tunes. In the Hamiltonian theory the chromaticity of beta-functions does not come from energy-dependent focusing strength of quads (as one would intuitively expect) but from drift spaces where the transverse momentum is large (low-beta regions). The symplectic transformations for that are:

$$
\begin{aligned}
X & =X-L \cdot X^{\prime} \cdot \frac{\Delta E}{E} \\
Y & =Y-L \cdot Y^{\prime} \cdot \frac{\Delta E}{E} \\
Z & =Z-L \cdot\left(X^{\prime 2}+Y^{\prime 2}\right) / 2
\end{aligned}
$$

where L is the "chromatic drift" length. Then, we have to adjust the betatron tune chromaticities which are also affected by "chromatic drift". For that, we use an artificial element (insertion) with the following Hamiltonian:

$$
H=I_{x} \cdot\left(2 \pi \nu_{x}+C_{x} \frac{\Delta E}{E}\right)+I_{y} \cdot\left(2 \pi \nu_{y}+C_{y} \frac{\Delta E}{E}\right),
$$

where $C_{x}$ and $C_{y}$ are the [additions to the] chromaticities of betatron tunes. 


\section{Diffusion and Noise}

Diffusion and noise are simulated by a single random kick applied to the macroparticles once per turn. Strength of the kick on different coordinates is given by a symmetrical matrix representing correlations between Gaussian noises. In the Tevatron, the diffusion is rather slow in terms of the computer simulation - the characteristic time for the emittance change is around an hour, or $\sim 10^{8}$ turns. In order to match the diffusion and the computer capabilities, the noise was artificially increased by three orders of magnitude. We justify this approach in [3].

\section{PROGRAM FEATURES}

Since the beam-beam code uses the "weak-strong" model, it can be very efficiently parallelized. Each processor tracks its own set of particles and the nodes need to communicate very rarely (at the end of each step), just to gather the obtained statistics. Hence, the productivity grows almost linearly with the number of nodes and in the case of the Tevatron ( 2 main IPs plus 70 PCs) it reaches $\sim 10^{10}$ particles $\times$ turns/day on a 32-node cluster of Pentium-IV $1.8 \mathrm{GHz}$ processors.

There are also two auxiliary GUI codes. The first one automates production of the LIFETRAC input files for different bunches from the OptiM output files. The second one is dedicated for browsing the LIFETRAC output files and presenting the simulation results in a text and graphical (histogram) form.

\section{DISCUSSION}

The developed set of programs can address multiple issues of simulation of beam-beam effects in the Tevatron. We estimate the inaccuracy of the present beam measurements as about $20 \%$ in betatron function. The code reproduces linear part of the problem very well. For example, the simulated bunch-by-bunch orbits match the measured ones (Fig. 2,3). The significance of the PCs is illustrated in

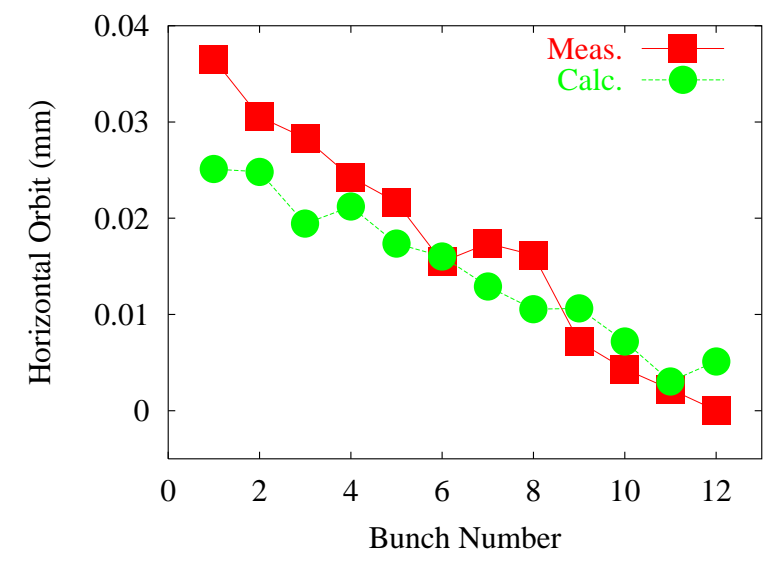

Figure 2: Horizontal antiproton bunch orbit measured by synchrotron radiation monitor (squares, store \#3530) and simulated using LIFETRAC (circles).

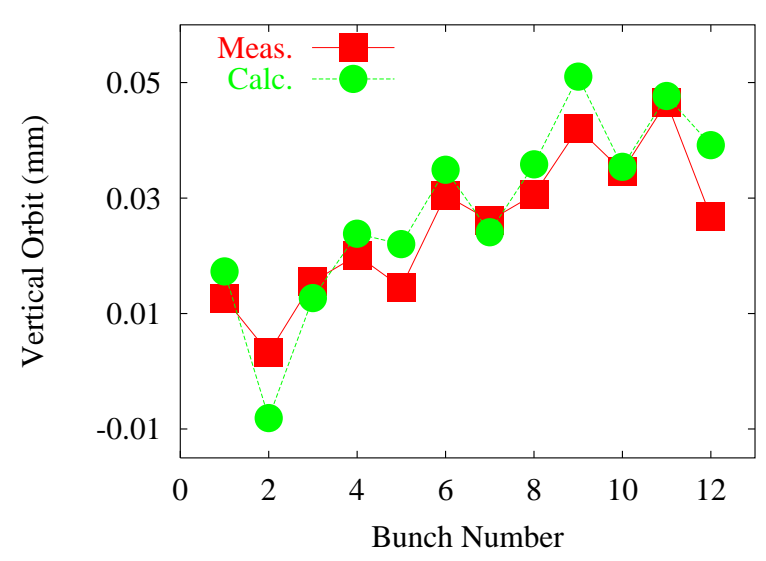

Figure 3: Vertical antiproton bunch orbit measured by synchrotron radiation monitor (squares, store \#3530) and simulated using LIFETRAC (circles).

Fig. 4, where a bunch intensity is plotted vs. time $\left(2 \times 10^{6}\right.$ turns correspond to about 15 hours in the Tevatron) with the complete set of IPs and PCs, and with PCs turned off. It is clear that PCs dominate the particle losses.

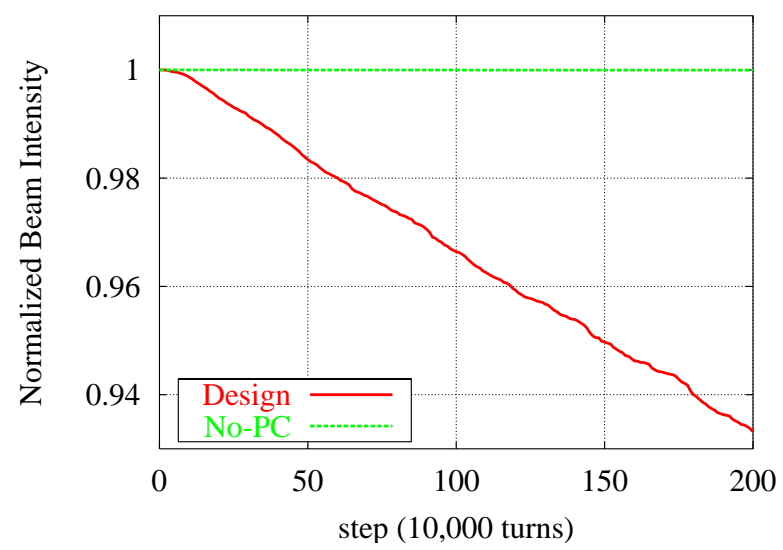

Figure 4: Normalized intensity of bunch \#6 simulated in the presence (solid line) and in the absence (dashed line) of Parasitic Collisions.

Various aspects of beam-beam effects in the Tevatron are discussed in [3].

\section{REFERENCES}

[1] V.Shiltsev, et al., TPAP032, these Proceedings

[2] V.Sajaev, et al., MPPE065, these Proceedings

[3] A.Valishev, et al., TPAT083, these Proceedings

[4] D. Shatilov, Part. Accel. 52, 65 (1996)

[5] K.Hirata, H.Moshammer, F.Ruggiero, KEK Report 92-117, 1992

[6] V.Lebedev, http://www-bdnew.fnal.gov/ pbar/organizationalchart/lebedev/OptiM/optim.htm

[7] V.Lebedev, S.Bogacz, http://www.cebaf.gov/ lebedev/AccPhys/ 